\title{
A GENERALIZED FORCE AND CHIP FLOW MODEL FOR OBLIQUE CUTTING AND VARYING UNDEFORMED CHIP CROSSSECTIONS
}

\author{
L. Meier ${ }^{1 *}$, L.Seeholzer ${ }^{1}$, K. Wegener ${ }^{1}$ \\ ${ }^{1}$ Institute of Machine Tools and Manufacturing (IWF), ETH Zurich, Switzerland \\ *Corresponding author; e-mail: meier@iwf.mavt.ethz.ch
}

\begin{abstract}
Simple cutting force models are well suited for orthogonal cutting. However, industrial processes often use oblique cutting with complicated cutting edge shapes, where simple cutting force models underestimate the forces. The new cutting force model is based on an existing model for restricted chip motion, but generic cutting edge and rake face shapes and arbitrary process kinematics are accepted to calculate the chip flow direction. The model is able to predict the force changes in the beginning of a turning process, when a tool with large nose radius enters the workpiece, and the chip movement in drilling.
\end{abstract}

Keywords:

Cutting forces; Restricted chip motion; Turning; Drilling

\section{INTRODUCTION}

Cutting processes are involved in the production of many industrial goods and products of the daily life. In order to increase the quality and to reduce the costs of those parts, cutting processes have to be optimized with respect to part quality and economy. The tool itself, which is represented by the macro- and micro-geometry, the substrate and the coating are usually optimized by the tool manufacturer. The process, i.e. the cutting speed, the feed rate and the toolpath, is usually optimized by the tool user. These steps require highly experienced specialists. Models of the cutting process can help them to achieve better solutions.

The process forces in cutting determine the necessary power which has to be delivered by the machine. They lead to deflections of the tool, workpiece and machine which in turn lead to deviations of the target geometry. Furthermore, they have an influence on surface integrity and burr formation and can cause chattering. The mechanical loading of the cutting edge is one of the main factors promoting wear. Therefore, real time cutting force measurements are gaining industrial interest as toolcondition monitoring equipment [Byrne 1995, Hidayah 2015]. In order to maximize the yield of cutting force data, cutting force models are needed.

\section{STATE OF THE ART}

\subsection{Analytical and empirical cutting force models}

As one of the first researchers, Merchant [Merchant 1945] investigated cutting processes with defined cutting edges and developed an analytical cutting force model for orthogonal cutting. He assumed a constant shearing stress in the infinitely thin, straight shear plane and applies the coulomb friction model. Furthermore, the tool is assumed to be infinitely sharp with no friction on the flank face. The cutting force and thus the cutting power reaches a minimum value at a certain angle of the shear plane. Merchant assumed this value to be the actual shear plane angle observed in cutting. Despite its very limiting assumptions, the model is still used today, for example to estimate the coefficient of friction for new tool types [Grzesik 2013]. Merchants model leads to cutting forces which are proportional to the undeformed chip thickness. In reality, the forces show a less than proportional increase with chip thickness. Kienzle [Kienzle 1952] used an empirical approach and suggested a power law with an exponent between 0 and 1 for the undeformed chip thickness. Kienzle's model is intensively used today, as its available for cutting force predictions in many CAM tools and myriads of measuring data are available and tabulated. An even simpler approach is presented by Budak and Altintaş [Budak 1996], commonly referred to as the Altintaş model. This model separates the cutting force into a constant portion caused by the cutting edge, which is not affected by the depth of cut, and a variable portion, which is proportional to the local undeformed chip thickness.

When a straight, oblique cutting edge with an inclination angle is used, the chip will not flow orthogonally to the cutting edge. Stabler [Stabler 1951] suggest that the chip flow deviates from the orthogonal direction with the same angle $\eta_{c}$ as the inclination angle of the cutting edge $\lambda_{S}$, being referred to as Stabler's law. Later studies confirm this tendency but obtain values up to $20 \%$ smaller than the inclination angle due to friction effects on the rake face [Orra 2018]. The deviations of the chip flow direction from Stabler's law at higher inclination angles are explained by Moufki et al. [Moufki 2000] with a temperature dependent coefficient of friction and a viscoplastic material model.

Considering an oblique cutting tool, the rake angle can be defined in three different ways [Brown 1964]: 
- the normal rake angle, measured in the too orthogonal plane, a plane orthogonal to the cutting edge

- the velocity rake angle, measured in the tool working plane, a plane parallel to the cutting velocity and orthogonal to the machined surface

- the effective rake angle, measured in a plane parallel to the chip flow direction and orthogonal to the machined surface

Brown et al. [Brown 1964] performed cutting tests with varying obliqueness and found that the normal rake angle is the most significant rake angle in terms of chip formation mechanism and cutting force.

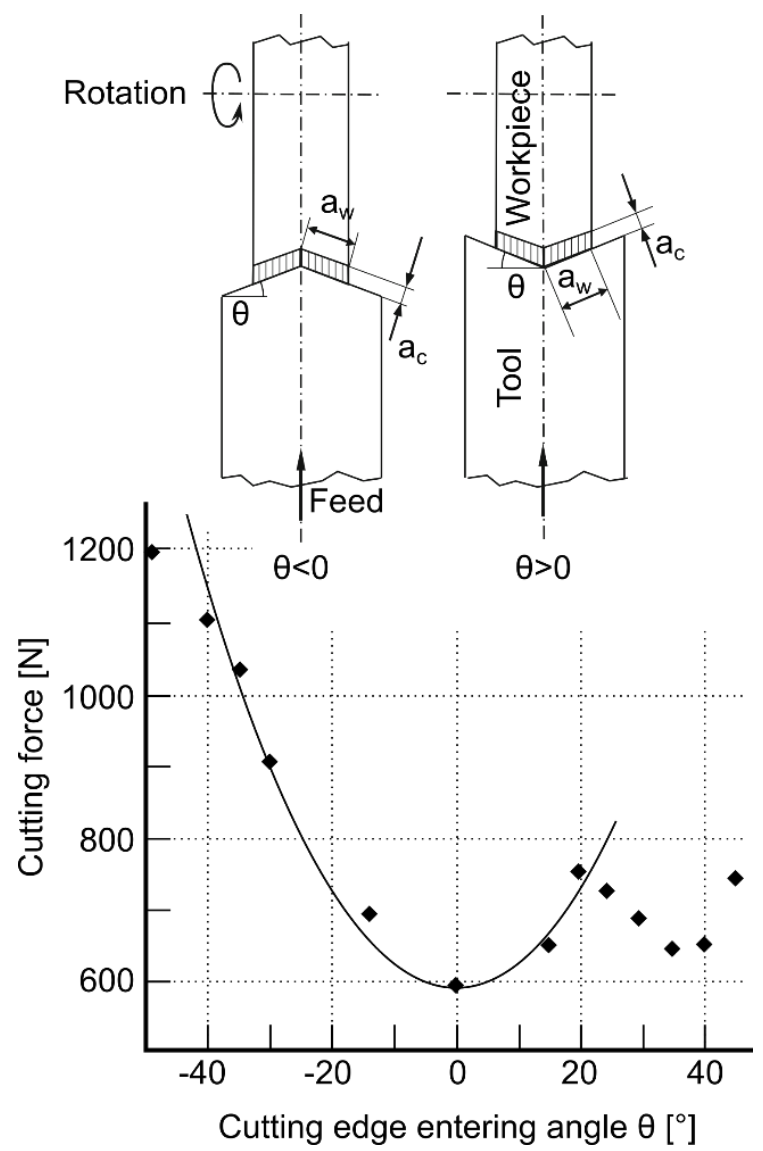

Fig. 1: Top: experimental setup to measure the force increase through restricted chip motion. Bottom: experimental data. Modified after [Shi 2018].

Shi et al. [Shi 1999, Shi 2018] studied the influence of confined chip movement. They used symmetrical cutting tools with both positive and negative entering angles as shown in the top of Fig. 1. In the case of $\theta<0$, if two independent chips formed on every side of the cutting tool, they would cross each other in the middle. However, as a single chip is formed, compressive stresses in the chip force both sides of the chip to move parallel to the feed direction. This restriction in movement causes higher cutting forces. The same effect could be observed in the case of $\theta>0$. If two independent chips formed, they would flow apart from each other. However, the chip is held together by tensile stresses, forcing the chip to move straight in feed direction, again causing an increase in cutting force. For $\theta>0$ the effect is limited to values below $20^{\circ}$, presumably because the chip breaks apart in the middle, leaving two noninterfering chips at higher angles $\theta$. The feed has been increased and the workpiece width has been decreased in the tests with higher absolute values of $\theta$ in order to keep the undeformed chip thickness $a_{c}$ and width $a_{w}$ constant. A simple empirical equation for the force increase is presented [Shi 2018]:

$F_{c}=F_{c 0} *\left(1+0.76 \theta^{2}\right)$

With $F_{c}$ being the cutting force, $F_{c 0}$ being the orthogonal cutting force and $\theta$ being the entrance angle in radians.

A force increase is also observed if the chip locally is not able to move with the optimal velocity, but the influence of restricted chip speed is much more difficult to test. Shi [Shi 2018] used a tool stack with different rake angles with all tools being engaged at the same time and forming a single chip. Higher rake angles lead to less chip compression and therefore to a higher chip speed. By stacking a tool with high rake angle between two tools with low rake angles, the chip of the middle tool is forced by shear stresses in the chip to move at a lower speed than in the unrestricted case, whereas the chip parts of the outer segments are forced to move faster than in the unrestricted case. By using different combinations of rake angles, the effect of the speed restriction can be isolated. It turns out that a similar relation as in Eq. 1 is valid.

\subsection{FE cutting force models}

In recent years, numerical models gained interest, not least due to the more affordable computational power. The finiteelement analysis (FEA) is the most commonly used numerical method for partial differential equations [Davim 2008]. A comprehensive review on the recent developments in FEA for cutting simulation is presented by Lauro et al. [Lauro 2015]. FEA is less restricted in terms of geometry of the cutting tool and kinematics of the process than the analytical models. Usually, however, more complex material and friction models need to be employed to achieve a correct representation of the material behavior, leading to extensive studies to determine the parameters. Furthermore, the computational costs are higher than that of analytical and empirical models, leading to computation times in the range of hours to days, making it unsuitable for multiparameter optimization.

Nevertheless, FEA software tools are commercially available and are used industrially by tool manufacturers.

\subsection{Cutting force measurement}

Cutting forces are usually measured with a piezoelectric dynamometer or with strain gauges on the tool or the workpiece side. While the steady state total forces are easy to measure, the assessment of the local force distribution requires additional experimental considerations.

The distribution of the thrust force and the force tangential to the cutting speed causing drilling torque can be determined by assuming that the total force is determined from a superposition of independent cutting processes along the cutting edge. The local distribution of the forces can then be determined by drilling into material predrilled with a smaller diameter. This method is often applied to eliminate the influence of the chisel edge [Boeira 2011].

The radial component of the drilling force is not observable in conventional drilling with a symmetrical drill, as the components from every cutting edge cancel each other out. Several methods to overcome this problem exist:

A drill with a single cutting edge can be used. Such a drill tends to be unstable. Therefore, the drill is usually replaced with a stiffer turning tool on a lathe with the same geometry [Boeira 2011].

Another possibility is to drill with the drill axis on the edge of the workpiece, creating an open half hole, so that at any given time only one cutting edge is engaged. The forces can be measured in the workpiece. The asymmetrical 
forces lead to a deflection of the drill, limiting this method to the application for short and stiff drills.

In order to eliminate deflection, Schütte [Schütte 2014] placed a second workpiece with a small gap next to the workpiece on the dynamometer as shown in Fig. 2. In this way, deflections of the drill are eliminated but the axial, radial and thrust force are still measurable with a single setup. This setup can also be combined with the predrill technique to obtain a locally resolved distribution of the process force.
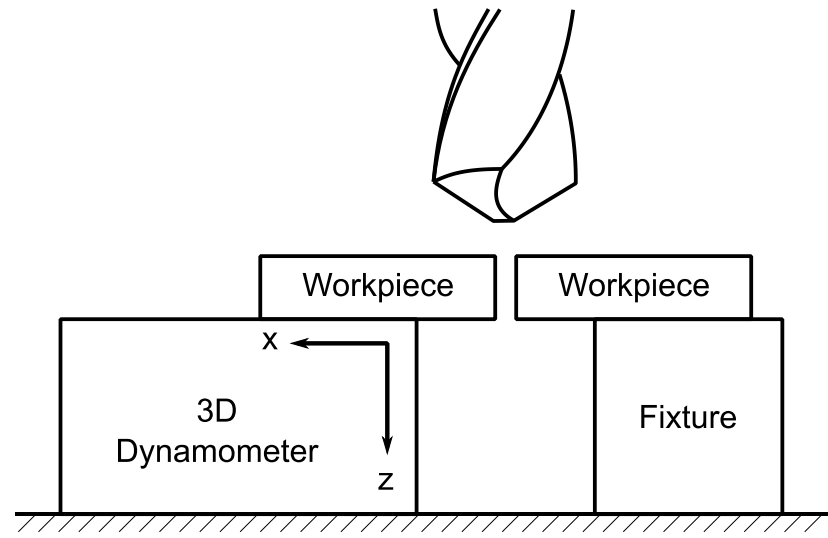

Fig. 2: Setup from Schütte [Schütte 2014] to measure the radial force components.

\section{UNFREE CUTTING FORCE MODEL}

The unfree cutting model by Shi [Shi 2018] is adapted to accommodate for generic cutting tool shapes, whereas Shi only investigated flat rake faces. Furthermore, a chip rotation around an axis perpendicular to the rake face is allowed as additional degree of freedom and transient engagement conditions are considered.

\subsection{Geometry}

The cutting edge is divided into small linear segments. The geometry of the rake face has to be provided as a list of points of the cutting edge with a corresponding vector being part of the rake face. The local cutting edge direction is calculated as the difference between two adjacent points on the cutting edge. If the provided vector being part of the rake face is not perpendicular to the cutting edge, it is rotated around the cross product of the rake face vector and the local cutting edge direction (i.e. the local normal vector of the rake face), until it is perpendicular to the cutting edge. Only the linear part of the rake face in the immediate vicinity of the cutting edge is considered. The rake face is flattened. The local part of the rake face is always developable in a mathematical sense, as this part of the cutting edge can only be single-curved. The rake face is reconstructed by joining each rake face segment in a plane (see Fig. 3, gray line). Note that this operation increases the nose radius and the corner angle of any non-planar rake face.

\subsection{Process kinematics}

The cutting speed for each segment is calculated based on the process kinematics. For processes with a rotational cutting movement, the angular velocity is multiplied with the distance of the cutting edge from the rotational axis. In the case of turning or drilling, the undeformed chip crosssection area is calculated as the intersection between the insert shape projected in the direction of the cutting speed and the current workpiece surface shape. The current workpiece surface in turn corresponds to the contour of the cutting edge after the last cut as shown in Fig. 3 . In a next step, the local chip load is calculated by dividing the undeformed chip cross-section area into slices orthogonally to the cutting edge as shown in Fig. 6, c). The area of each slice assigned to be the chip load of the corresponding cutting edge segment.

The angle between each cutting edge segment and the local cutting speed is calculated. The difference of this angle to $90^{\circ}$ corresponds to the local inclination angle or the obliqueness.

\subsection{Chip flow}

Stabler's law [Stabler 1951] is assumed for the unrestricted chip flow direction of each segment, i.e. the chip flow direction is assumed to deviate from the direction orthogonal to the cutting edge by the local obliqueness angle (see Fig. 3, red arrows). After its formation, the chip is assumed to behave as thin shell element with infinite tensile stiffness in both directions but zero bending stiffness in both directions. The chip therefore adapts to the rake face and the chip motion can be studied in the flattened view of the rake face. In the flattened view, the chip behaves like a rigid body with three degrees of freedom: two lateral motion components and a rotation around the orthogonal direction to the rake face.

The three chip motion components are determined by an optimization routine. The deviation from the unrestricted chip flow of each cutting edge segment causes an increase in the cutting force, which is proportional to deviation squared, as shown by Shi [Shi 2018]. The increase is described with two different proportionality constants, one for angle and one for speed deviations. Based on the process kinematics, the chip can have an uneven thickness. Thicker parts of the chip are assumed to have a bigger influence on the chip direction and velocity than the thinner parts. Therefore, the chip load, i.e. the chipping area belonging to each segment is used as a weight in the optimization routine. This problem is linear in parameters and therefore leads to a weighted least squares optimization, which is solved by linear regression.

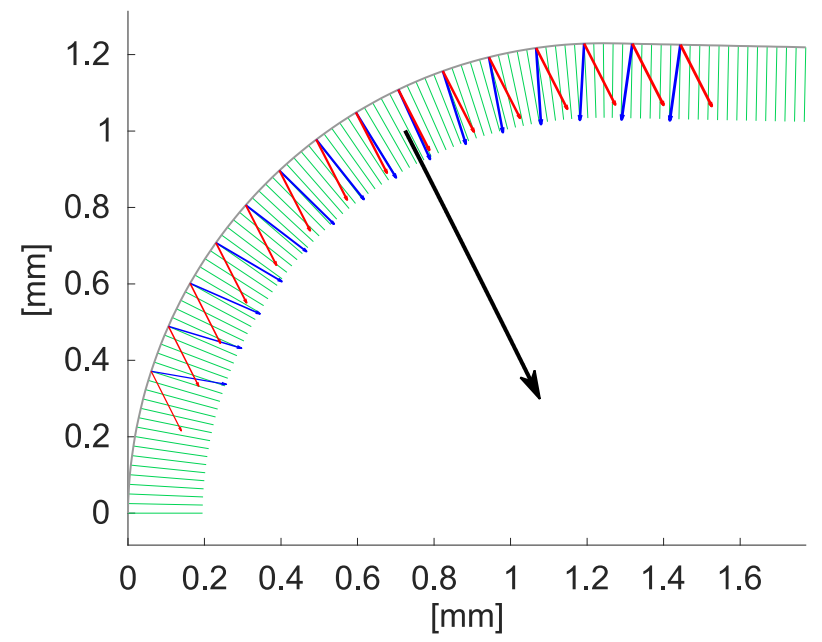

Fig. 3: Unwrapped insert rake face example for the situation shown in Fig. $6 \mathrm{c}$ ). The cutting edge is shown in gray. Normal vectors of each segment are shown in green.

Blue arrows indicate the unrestricted chip flow. Arrow

thickness indicates the local chip load. The black arrow indicates the movement of the chip centroid. Red arrows show the rigid body movement in every segment.

From the optimal chip motion parameters, speed and direction indicated in Fig. 3 by the black arrow, the chip motion angle and speed for each point on the cutting edge is calculated (see Fig. 3, blue arrows). 


\subsection{Cutting force}

The cutting force is composed of four components. The force caused by the chip load is oriented perpendicular to the rake face of each element. It is proportional to the chip load with a constant $k_{A}$, to the angular deviation of chip movement from the unrestricted direction squared with a constant $k_{u f}$ and to the deviation from the unrestricted velocity with a constant $i_{u f}$ :

$d F_{\text {Chip }}=k_{A} \cdot d A\left[1+k_{u f}\left(\theta-\theta_{u . r .}\right)^{2}+i_{u f}\left(v-v_{u . r .}\right)^{2}\right]$

with $d A$ being the undeformed chip cross-section area corresponding to each respective segment, $\theta$ the local chip flow angle determined with the optimization routine in the previous step, $\theta_{u . r}$ the unrestricted chip flow angle from Stabler's law, $v$ the chip velocity determined with the optimization in the previous step and $v_{u . r}$. the unrestricted chip flow speed derived from the process kinematics. The chip compression is included in the constant $i_{\text {u.f. }}$.

The force caused by friction on the rake face is calculated using Coulomb friction. The coefficient of friction $\mu$ is used to calculate the friction force from the chip load force $d F_{\text {Chip }}$ :

$d F_{\text {Fric }}=\mu \cdot d F_{\text {Chip }}$

To determine the correct orientation of the frictional force component in the 3D space, the vector orthogonal to the cutting edge lying in the rake face determined in the geometry definition step is rotated around the vector normal to the rake face by the angle $\theta$.

The effect of the cutting edge micro-geometry is considered by adding a constant term to the process force for each engaged segment. The component responsible for a higher cutting force acts in the plane containing the cutting speed and the cutting edge, orthogonally to the cutting edge:

$d F_{\text {edge }, c}=d L \cdot k_{\text {edge }, c}$

with $d L$ being the cutting edge length belonging to a segment and $k_{\text {edge, }}$ being a constant.

The last component is a constant force acting perpendicular to the cutting speed and the cutting edge:

$d F_{\text {edge }, c}=d L \cdot k_{\text {edge,c }}$

This component mainly contributes to the feed and passive force.

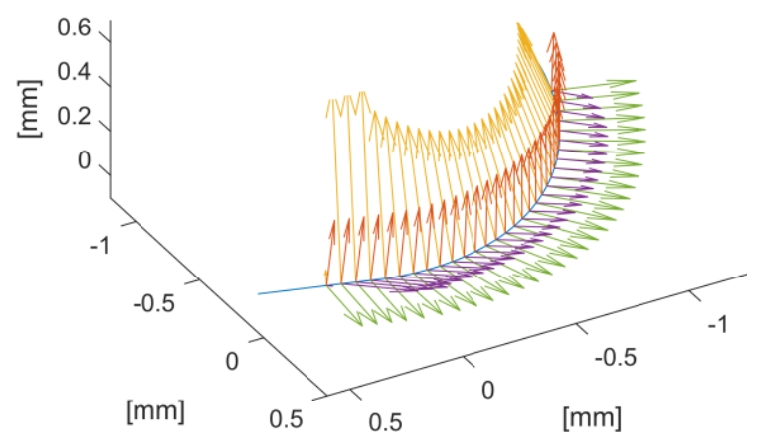

Fig. 4: The four local force components. $d F_{\text {Chip }}$ is shown in yellow. $d F_{F r i c}$ is shown in violet. $d F_{\text {edge,c }}$ is shown in orange. $d F_{\text {edge,f }}$ is shown in green. The length of the arrows corresponds to the force value. For better visibility, the forces are shown in the direction they act on the workpiece.

The four force components for each element shown in Fig. 4 are summed up to get the 3D process force for a given engagement geometry. By projecting this force in the cutting, feed and passive direction, forces comparable to the experimental data are obtained. By repeating this procedure for different engagement situations during the transient period at the process start, the forces as a function of time can be calculated as shown in Fig. 6 .

\section{EXPERIMENTAL SETUP}

\subsection{Turning test}

The model is tested in an internal face turning setup. A piezoelectric cutting force dynamometer Kistler 9129AA is mounted on the turret of a lathe. A boring bar is then mounted onto the dynamometer. An insert of type Sandvik Coromant CNMG 1204 12-SM 1125 is clamped at an inclination angle of $-6^{\circ}$ and an orthogonal rake angle of $10^{\circ}$. The negative insert has a rhombic shape with a corner angle of $80^{\circ}$ and is clamped at a lead angle of $5^{\circ}$. The corner radius is $1.2 \mathrm{~mm}$. The insert has a simple circumferential chip breaker with an angle of $15^{\circ}$ (see

Fig. 5).

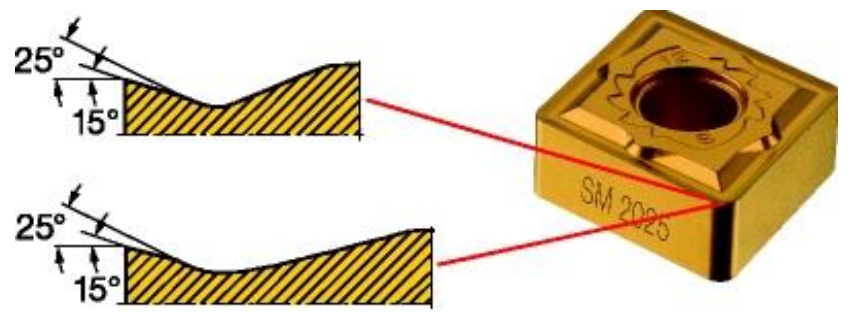

Fig. 5: Insert used in the turning tests [Sandvik 2019]

a)

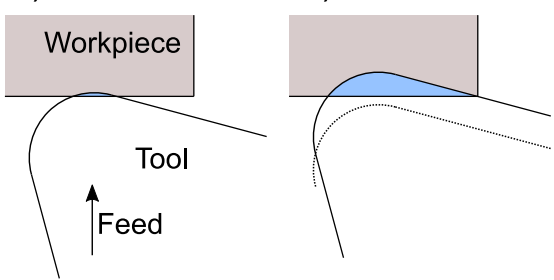

c)

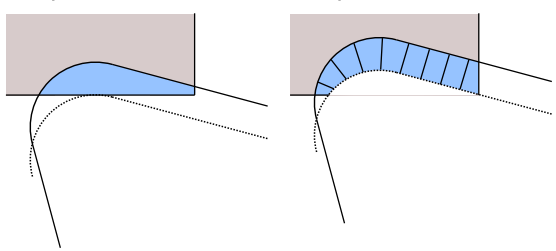

e)

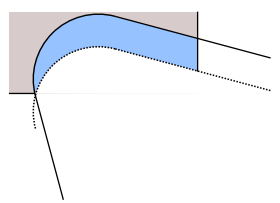

Fig. 6: Undeformed chip cross-section area (light blue) at different positions of the cutting tool in turning. The position of cutting edge in the last revolution is indicated with a dashed line. The segments used in the model are indicated in d). Not to scale.

An internal transverse face turning process is carried out on a Ti6Al4V workpiece by starting in a preturned hole with diameter $276 \mathrm{~mm}$. The cutting speed is set to $50 \mathrm{~m} / \mathrm{min}$, the feed per revolution is $0.28 \mathrm{~mm}$ and the depth of cut is $1.5 \mathrm{~mm}$. The test is stopped after a diameter of $277.8 \mathrm{~mm}$ is reached. The process can be divided into six stages, with the sixth stage being the steady state, which is not investigated. In the first stage, only the insert corner radius is engaged (before Fig. 6 a). After that, the workpiece is still 
cylindrical, but the straight part of the main cutting edge is engaged. Then, the straight part of the cutting edge reaches the workpiece corner (Fig. 6, b). After one revolution (Fig. $6, \mathrm{c})$, the surface of the workpiece already has a groove from the previous revolution. Then, the groove reaches the workpiece edge. (Fig. 6, d). Finally, the undeformed chip cross-section area reaches a steady-state (Fig. 6, e), with constant forces.

The signal is sampled at a rate of $1111 \mathrm{~Hz}$ and filtered with a fourth order Butterworth filter with a cut-off frequency of $22 \mathrm{~Hz}$.

\subsection{Drilling test}

Drilling force tests are performed with the setup proposed by Schütte [Schütte 2014] (see Fig. 2). Two strips of aluminium ENAW 7175 are fixed with a space of $2.2 \mathrm{~mm}$ next to each other to prevent the chisel edge from cutting. A $6.8 \mathrm{~mm}$ conventional HSS drill with TiN-coating and a tip angle of $118^{\circ}$ is used with $4511 \mathrm{rpm}$ and a feed of 0.1 per tooth.

The first harmonic frequency of the dynamometer setup is close to the tooth engagement frequency Therefore the force signal cannot be resolved locally for each position of the cutting edges. Instead, the signal is filtered with a fourth order Butterworth filter with a cut-off frequency of $57 \mathrm{~Hz}$. The aluminium workpiece is assumed to be isotropic and the feed motion during a half revolution is neglected, leading to the assumption that the process forces are constant during a half rotation of the drill. Further assuming the system behaves linearly, the tangential and radial forces can be integrated over half a revolution and averaged as shown in Fig. 7, leading to the following relations:

$F_{\text {tan }}=\frac{\pi}{2} \cdot \overline{F_{y}}$

$F_{\text {rad }}=\frac{\pi}{2} \cdot \overline{F_{x}}$

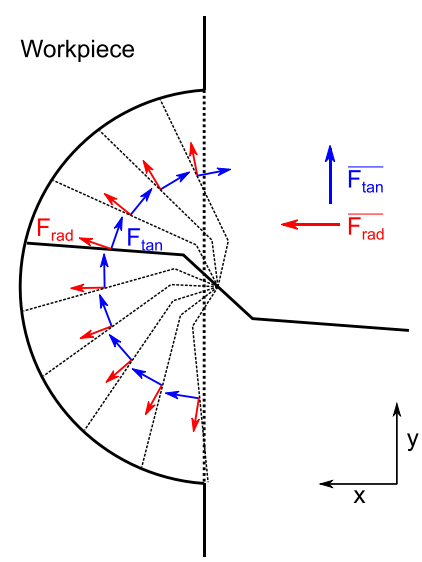

Fig. 7: Averaging the tangential and radial force over half a revolution.

The drilling process can be divided into four stages. In the first stage, the workpiece still has the original shape. The undeformed chip cross-section area increases with the square of the feed. After half a revolution the situation in Fig. 8 a) is reached. After this point, the chip area only increases linearly. As soon as the drill edge reaches the workpiece in Fig. 8 b), the undeformed chip cross-section area only increases marginally until reaching the steady state in Fig. $8 \mathrm{c}$ ).

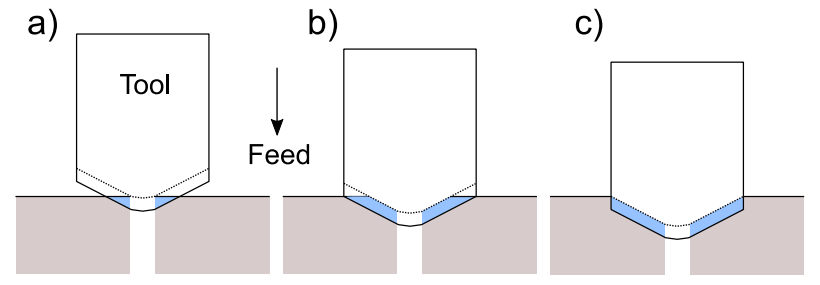

Fig. 8: Undeformed chip cross-section area (light blue) at different positions of the cutting tool in drilling. The position of cutting edge in the last half revolution is indicated with a dashed line. Not to scale.

\section{SIMULATION}

\subsection{Turning}

For the simulation of the process forces in the beginning of a turning process, the geometry of the rake face is programmed analytically, as the inserts' rake face is consisting of cones and planes in this case. The cutting edge is divided into 200 segments with equal length. The tool path is divided into steps of $20 \mu \mathrm{m}$. A total tool path of $1.5 \mathrm{~mm}$ is simulated.

As far as possible, the necessary parameters are determined from literature. The coefficient $k_{A}$ linking the undeformed chip cross-section area with the force acting on the rake can be determined from orthogonal cutting force data, e.g. provided by Wyen et al. [Wyen 2010]. The coefficient is determined as the slope of the force as a function of the undeformed chip thickness at undeformed chip thicknesses much bigger than the cutting edge radius divided by the cutting width. In this case, resulting in $1000 \mathrm{~N} / \mathrm{mm}^{2}$.

Different researchers measured the coefficient of friction $\mu$ between cutting tools and titanium. The published values are around 0.5 [Hong 2001], around 0.3 [Wyen 2010] and around 0.3 [Egaña 2012]. A value of 0.37 is assumed as the mean value of the three sources.

The coefficients for the force increase due to chip flow restriction are determined by inverse fitting in a preliminary experiment. With 0.9 for $k_{u . f}$. and 1.8 for $i_{u . f}$, they are slightly higher than the ones determined in steel cutting by Shi [Shi 2018]

The coefficients $k_{e d g e, c}$ and $k_{e d g e, f}$ cannot be determined from literature, as they depend on the cutting edge microgeometry. If most accurate results are seeked, they even have to be determined separately for each cutting edge as two cutting edges never have the exact same shape. In this case, the coefficients $k_{\text {edge,c }}$ and $k_{\text {edge,f }}$ are determined by inverse fitting to experimental data from 10 trials. On average, the optimal $k_{e d g e, c}$ is $81 \mathrm{~N} / \mathrm{mm}$ and the optimal $k_{\text {edge,f }}$ is $86 \mathrm{~N} / \mathrm{mm}$.

The resulting cutting forces are shown in Fig. 9. The first phase of the cutting process is characterized by a perfectly symmetrical cutting tool. This is however not visible, because the straight part of the main cutting edge engages already after $5 \mu \mathrm{m}$. At $22 \mu \mathrm{m}$, the main cutting edge reaches the workpiece edge as depicted in Fig. 6 b), leading to the first visible kink, as the undeformed chip cross-section area does only increase to the left and in depth, but not to the right side anymore. At a tool path length of $0.28 \mathrm{~mm}$, which corresponds to the feed per revolution, as in Fig. $6 \mathrm{c}$ ), a second kink is visible. The transition from the fourth to the fifth phase is not visible. 


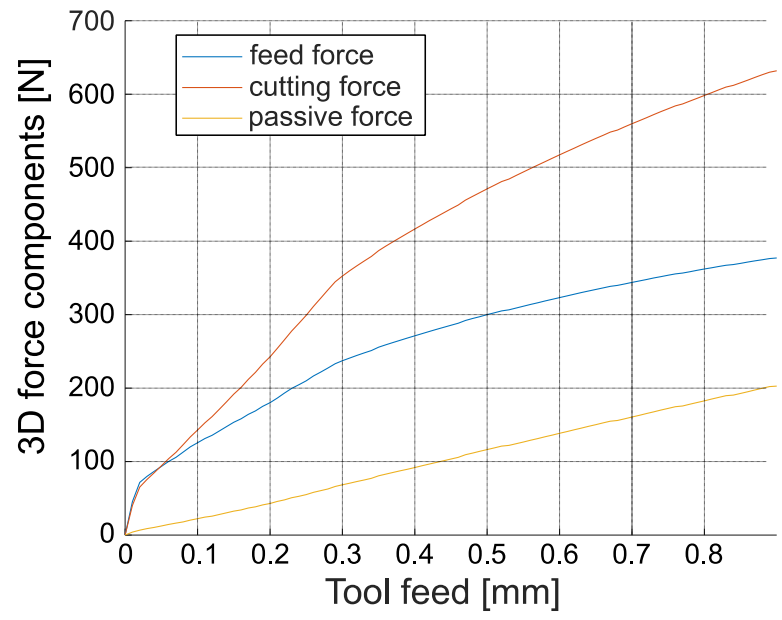

Fig. 9: Simulation result of the cutting forces in the beginning of the turning process.

\subsection{Drilling}

The main cutting edge of the drill is divided into 139 segments. The drill has a straight cutting edge with a constant rake angle of $20^{\circ}$. Therefore the developed view of the rake face shown in Fig. 10 is trivial.

The main cutting edge offset from a radial direction due to the chisel edge leads to an inclination angle, which in turn leads to the unrestricted chip motion vectors pointing outwards. Contrary to the situation in turning, the cutting speed varies significantly along the cutting edge, leading to a pronounced chip rotation. The center of rotation is not in the middle of the drill but is shifted towards the outer corner of the cutting edge due to the inclination angle.

The simulated process forces are increasing almost linearly as shown in Fig. 11, because the undeformed chip crosssection area is increasing almost linearly as well and chip flow restriction does not play a major role.

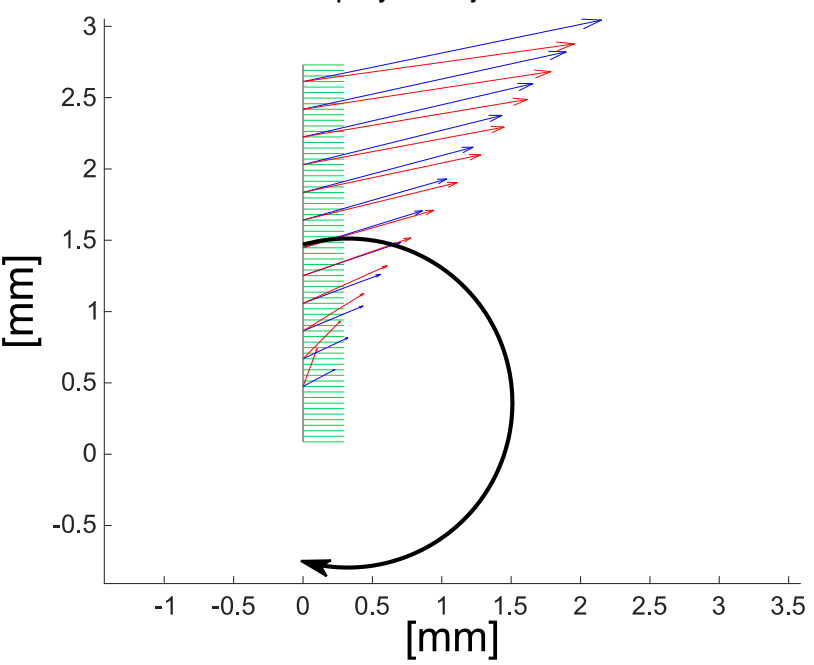

Fig. 10: Unwrapped insert rake face example for the situation shown in Fig. 8 b). The cutting edge is shown in gray. The transition point from the chisel edge to the main cutting edge is at the origin. Normal vectors of each segment are shown in green. Blue arrows indicate the unrestricted chip flow. The black arrow indicates the movement of the chip centroid. Red arrows show the rigid body speed at the position of every segment.

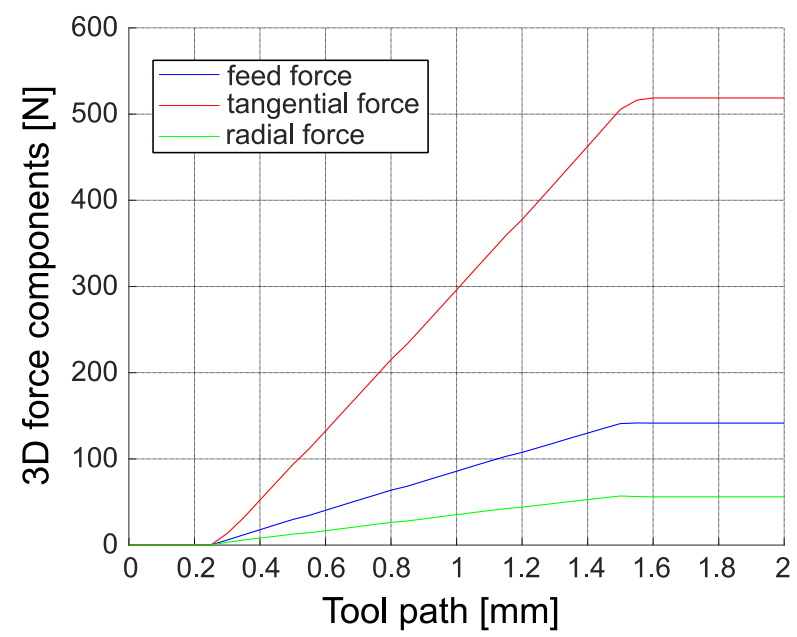

Fig. 11: Simulation result of the cutting forces in the beginning of the drilling process.

\section{VALIDATION OF THE MODEL}

The Kienzle model as well as the Altintaş model are not able to explain the increase in cutting force at the beginning of a turning process with a significant tool corner radius, which is over proportional to the undeformed chip cross-section area, as shown in Fig. 12. In the beginning, the undeformed chip cross-section is thin and wide, (see Fig. 6, a), leading to a smooth chip flow. Later in the process, the section is curved (see Fig. 6, d), leading to a chip flow restriction and an over proportional force increase.

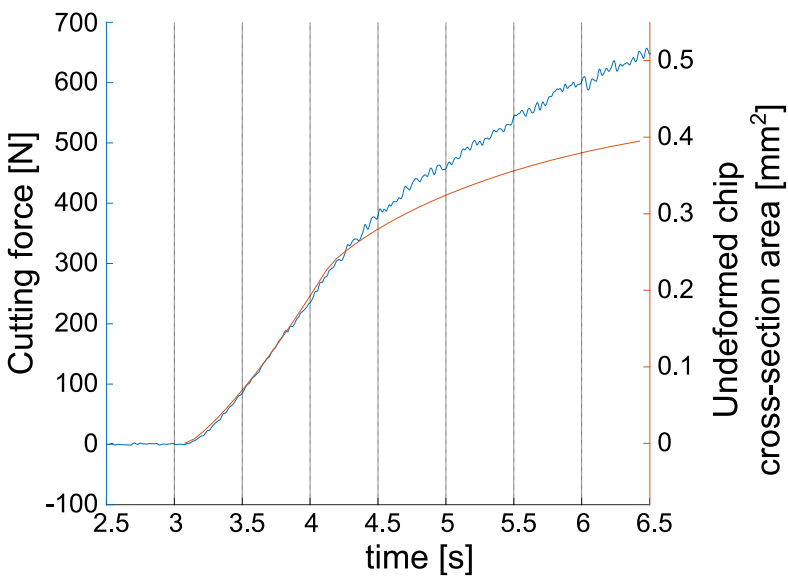

Fig. 12: Comparison between cutting force (blue) and undeformed chip cross-section area (red).

Wear can be excluded as a possible reason for the increase in force, as a second test with the same tool leads to the same forces.

The forces can be matched with an error of less than $10 \%$ (see Fig. 13). The absolute values are of course easy to obtain by fitting of the four unknown parameters, but the effects considered in the model determine the shape of the force curves. The predicted force shapes match the ones measured. Special features represented well by the model are the kink in the cutting force at $4.5 \mathrm{~s}$ and the almost linearly increasing passive force. 


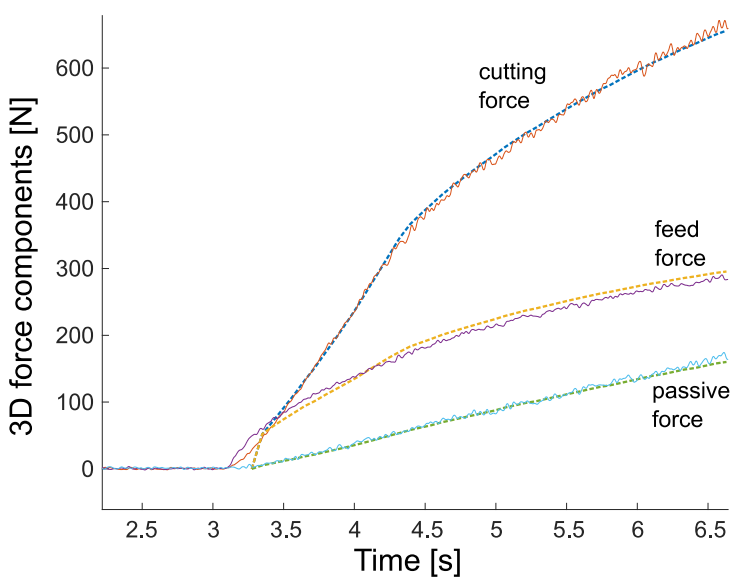

Fig. 13: Comparison between measured (solid) and modelled (dashed) forces in turning.

The biggest deviation from the measured forces occurs in the beginning. In this phase, no chip is formed, and the cutting tool is rubbing on the surface. This process as well as the elasticity of the tool are not considered in the modelling approach.

The measured forces deviate much more from the model than in turning (see Fig. 13), especially for the feed force. The differences may stem from vibration of the workpiece and tool, causing an engagement of the chisel edge, although the gap between the workpieces is set to the width of the chisel edge.

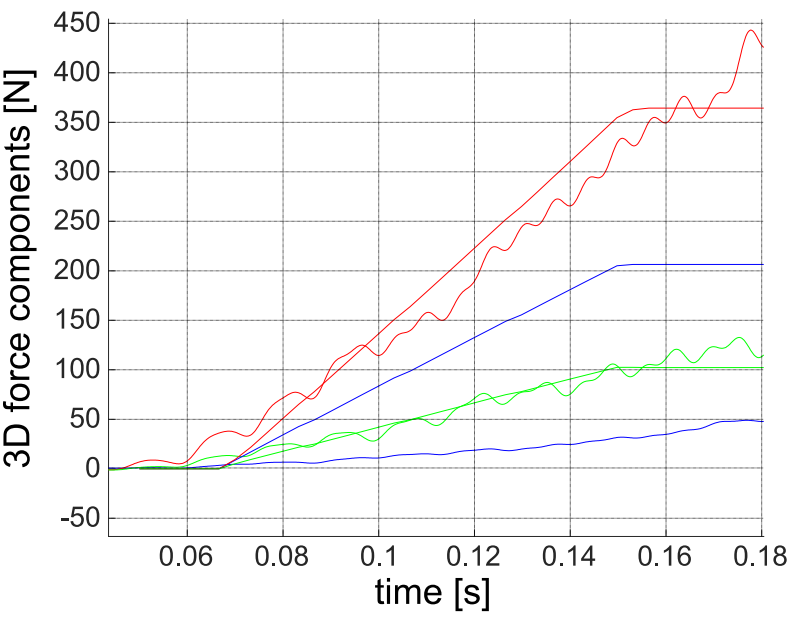

Fig. 14: Comparison between measured (solid) and modelled (dashed) forces in drilling. Red: tangential force, green: radial force, blue: feed force.

Fig. 15 shows the chips produced in the drilling process. The model is able to predict the curved shape. The model predicts a chip radius at the outer edge of $2.5 \mathrm{~mm}$. On the chips, a value of $3.2 \mathrm{~mm}$ is measured. The chips are wider than expected, meaning that the chisel edge contributed to the chip. The chisel edge is not considered in the model, explaining the difference between predicted and measured chip radius.

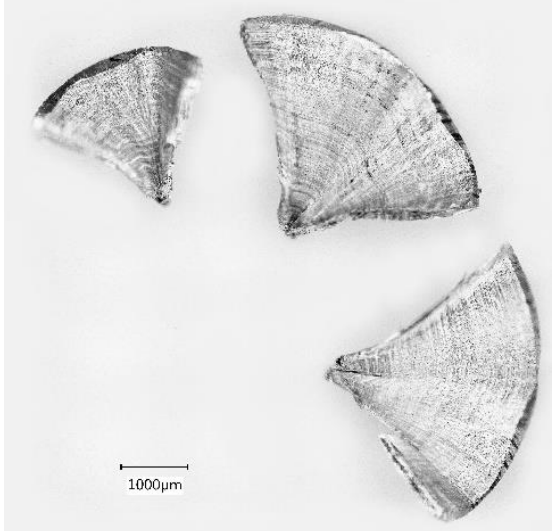

Fig. 15: Chips produced in the drilling process.

\section{CONCLUSION}

The introduced model presents a reasonable extension for simpler models which cannot cope with curved cutting edge geometries or chip breaker grooves. The model uses several decades less computational effort than an FE model and is therefore suited for quick optimization and inverse fitting.

The simpler models cannot explain the steep increase in cutting forces at the beginning of a turning process. By adding the term for restricted chip motion, the increase is matched. It is therefore very likely that the force increase is caused by the restricted chip motion, as wear could be eliminated as possible reason.

Drilling with conventional cutting edge shapes does not require the restricted chip flow model. The deviation from measured forces is up to a factor of four for the feed forces. In this case however, the chip motion itself is of great interest. The different cutting speeds along the cutting edge lead to a significant chip rotation in contrast to turning.

\section{OUTLOOK}

The model is tailored to the applications in turning and drilling processes. The influence of the rake angle is neglected in the presented model. Especially when cutting at a very high obliqueness, the effects described in literature have to be considered. The flank angle has an influence on the force $F_{\text {edge,f }}$, but the influence is overshadowed by more important effects in this setup. The influence of cutting speed is neglected as well, as this effect is only relevant in drilling, but could not be separated from other effects in this test setup. The friction model is very simple and there are more complicated and more accurate alternatives available.

The rubbing of the tool on the workpiece surface before cutting is neglected, because this effect is not relevant for the application.

The chip motion is only investigated close to the cutting edge, as this is the most important region for cutting forces. If the chip motion further away from the tool or even chip breakage is to be modelled, the chip shell with its associate motion data provided by the model can be used as an input.

The presented model provides a framework that can easily be extended, if necessary for a new application. Some effects can be studied in specially designed, simplified setups (e.g. as the setup in Fig. 1), other effects can be analytically derived and necessary parameters can be identified via fitting (e.g. as in this study). 


\section{ACKNOWLEDGMENTS}

The authors would like to thank Innosuisse - the swiss innovation agency for funding. Furthermore, the collaboration with Blaser Swisslube for the turning experiments is acknowledged.

\section{REFERENCES}

[Boeira 2011] Boeira A.M.G. et al. Contribution to the Modeling of Forces in Drilling with Twist Drills. Journal of Machine and Forming Technologies, 2011, Vol. 3, No. 1/2

[Brown 1964] Brown, R.H., Armarego, E.J.A. Oblique Machining with a Single Cutting Edge. Int. J. Mach. Too Des. Res., 1964, Vol. 4, pp. 9-25

[Budak 1996] Budak, E. et al. Prediction of milling force coefficients from orthogonal cutting data, Journal of Manufacturing Science and Engineering, 1996, Vol. 118, No. 2, pp. 216-224

[Byrne 1995] Byrne, G. et al. Tool Condition Monitoring (TCM) - The Status of Research and Industrial Application. Annals of the CIRP, 1995, Vol. 44, No. 2

[Davim 2008] Davim, P. Machining, Fundamentals and Recent Advances, 2008, Springer London. ISBN 978-184800-212-8

[Egaña 2012] Egaña, A. et al. Characterization of Friction and Heat Partition Coefficients during Machining of a TiAl6V4 Titanium Alloy and Cemented Carbide. Tribology Transactions, 2012, Vol. 55, No. 5, pp. 665-676

[Grzesik 2013] Grzesik, W. Zak, K. Friction quantification in the oblique cutting with CBN chamfered tools. Wear, 2013 , Vol. 304, pp. 36-42. ISSN: 0043-1648

[Hidayah 2015] Hidayah, M.T.N. et al. A Review of Utilisation of Cutting Force Analysis In Cutting Tool Condition Monitoring. International Journal of Engineering \& Technology, 2015, Vol. 15, No. 3, pp. 28-35

[Hong 2001] Hong, S. Y. et al. Friction and cutting forces in cryogenic machining of To-6Al-4V. International Journal of Machine Tools \& Manufacture, 2001, Vol. 41, pp. 2271 2285
[Kienzle 1952] Kienzle, O., Die Bestimmung von Kräften und Leistungen an spanenden Werkzeugen und Werkzeugmaschinen (in German). VDI-Z, 1952, Vol. 94 No. 11 , pp. 299-305

[Lauro 2015] Lauro, C.H. et al. Finite Element Method in Machining Processes: A Review. In: Modern Manufacturing Engineering, Davim J., 2015, Springer. ISBN 978-3-31920151-1

[Merchant 1945] Merchant, M.E. Mechanics of the metal cutting process. I. Orthogonal cutting and a type 2 chip. Journal of applied physics, 1945, Vol. 16, No. 5, pp. 267275

[Moufki 2000] Moufki, A. et al. Thermoviscoplastic modelling of oblique cutting: forces and chip flow predictions. International Journal of Mechanical Sciences, 2000, Vol. 42, pp. 1205-1232

[Orra 2018] Orra, K., Choudhury, S.K. Mechanistic modeling for predicting cutting forces in machining considering effect of tool nose radius on chip formation and tool wear land. International Journal of Mechanical Sciences, 2018, Vol. 142-143, pp. 255-268

[Sandvik 2019] Online tool catalog of Sandvik Coromant. www.sandvik.coromant.com. Accessed 24.5.2019

[Schütte 2014] Schütte, Ch. Bohren und Hobeln von kohlefaserverstärkten Kunststoffen unter besonderer Berücksichtigung der Schneide-Faser-Lage (in German). PhD-thesis, 2014, Technical University Hamburg-Harburg. ISSN: 1613-5244

[Shi 1999] Shi, Hanmin. Chip-ejection interference in cutting processes of modern cutting tools. Science in China Series E: Technological Sciences, 1999, Vol. 42, No. 3, pp. 275281.

[Shi 2018] Shi, H. Metal Cutting Theory, 2018, Springer. ISBN 978-3-319-73560-3

[Stabler 1951] Stabler, G.V., The Fundamental Geometry of Cutting Tools. Proceedings of the Institution of Mechanical Engineers, 1951, Vol. 165, No. 1, pp. 14-26

[Wyen 2010] Wyen, C.F., Wegener, K. Influence of cutting edge radius on cutting forces in machining titanium. CIRP annals, 2010, Vol. 59, No. 1, pp. 93-96 